\title{
Extending KIDs to the Mid-IR for Future Space and Suborbital Observatories
}

\author{
J. Perido ${ }^{1}$ D $\cdot$ J. Glenn ${ }^{1} \cdot$ P. Day ${ }^{2} \cdot$ A. Fyhrie ${ }^{1} \cdot$ H. Leduc ${ }^{2} \cdot$ J. Zmuidzinas ${ }^{3}$. \\ C. McKenney ${ }^{1}$
}

Received: 20 August 2019 / Accepted: 27 January 2020 / Published online: 14 February 2020

(c) The Author(s) 2020

\begin{abstract}
The galaxy evolution probe (GEP) is a concept for a probe-class space observatory to study the physical processes related to star formation over cosmic time. To do so, the mid- and far-infrared (IR) spectra of galaxies must be studied. These mid- and far-IR observations require large multi-frequency arrays, sensitive detectors. Our goal is to develop low NEP aluminum kinetic inductance detectors (KIDs) for wavelengths of 10-400 $\mu \mathrm{m}$ for the GEP and a pathfinder long-duration balloon (GEPB) that will perform precursor GEP science. KIDs for the lower wavelength range $(10-100 \mu \mathrm{m})$ have not been previously implemented. We present an absorber design for KIDs sensitive to wavelengths of $10 \mu \mathrm{m}$ shown to have around 75-80\% absorption efficiency through ANSYS HFSS (high-frequency structure simulator) simulations, challenges that come with optimizing our design to increase the wavelength range, initial tests on our design of fabricated $10 \mu \mathrm{m}$ KIDs, and theoretical NEP calculations.
\end{abstract}

Keywords Kinetic inductance detector $\cdot 10$ micron · Mid-infrared · Far-infrared · Astrophysics

\section{Introduction}

Observations of the mid- and far-infrared spectra of galaxies are a necessary means to explore the origins of galaxies, stars, and planets that make up the universe. From these spectra, the correlations of star-formation rates with other physical properties

J. Perido

joanna.perido@colorado.edu

1 Department of Astrophysics and Planetary Sciences, University of Colorado at Boulder, Boulder, CO 80309, USA

2 NASA Jet Propulsion Laboratory (JPL), Pasadena, USA

3 California Institute of Technology, Pasadena, USA 
can be investigated to expand our understanding of galaxy evolution. Galaxies have historically been studied through the emission features of dust and atomic fine structure lines in the far-IR. However, another known and studied region rich with information lies in the rest-frame emission features of polycyclic aromatic hydrocarbons (PAHs) with emission bands in the 3-13 $\mu \mathrm{m}$ range [1]. PAHs can be used to measure redshifts and characterize interstellar physical conditions and chemistry in millions of galaxies with a new infrared space observatory. The galaxy evolution probe (GEP) is a concept for a probe-class space observatory, with the goal to detect galaxies in these mid- and far-IR regions by utilizing large arrays of back-illuminated, lumped-element, microlens-coupled, sensitive aluminum kinetic inductance detectors (KIDs).

Although KIDs research is a popular and growing field, KIDs for detection at wavelengths from 10-100 $\mu \mathrm{m}$ have not been an area of particular emphasis. The investigation for KIDs at these wavelengths is also useful for other space observatories, such as the Origins Space Telescope. To address this, we investigate KID absorber designs capable of absorbing at GEP's shortest wavelength $(=10 \mu \mathrm{m})$ while achieving background limited sensitivity.

The GEP will have one instrument with two modules, an imager (GEP-I), and a dispersive spectrometer (GEP-S) consisting of 25,735 detectors and 24,640 detectors, respectively. The sensitivity requirements for the modules are a NEP of $1 \times 10^{-18} \mathrm{~W} / \sqrt{\mathrm{Hz}}$ for the GEP-I and $1 \times 10^{-19} \mathrm{~W} / \sqrt{\mathrm{Hz}}$ for the GEP-S. We also plan to demonstrate performance and raise the detector technology readiness level (TRL) with a pathfinder long-duration balloon (GEP-B) that will perform precursor GEP science. The KIDs on GEP-B will need a NEP on the order of $10^{-16} \mathrm{~W} / \sqrt{\mathrm{Hz}}$. The increase in the NEP when compared to the GEP is due to the (ambient) temperatures of the optical components on the GEP-B.

In this paper, we will present ANSYS HFSS ${ }^{1}$ (high-frequency structure simulator) software suite simulations of KID absorber designs capable of absorbing at $10 \mu \mathrm{m}$ and greater, preliminary measurements of the critical temperature of a fabricated test device and evidence of two-level system (TLS) noise, and theoretical NEP calculations for GEP.

\section{MID-IR KID Designs and HFSS Simulations}

The inductors will have the unit cell repeated in a circular envelope. This is shown in Fig. 1 Top where the geometry in Fig. 1 Bottom Left is repeated to form the absorber. To evaluate the absorption of the inductors at $10 \mu \mathrm{m}(v=30 \mathrm{THz})$, we used ANSYS HFSS. Simulations were done using a single unit cell simulated in an infinite periodic grid to approximate the repeated geometry across the absorber. A single unit cell is shown in Fig. 1 Bottom Right. Floquet ports were assigned to the faces of the vacuum and substrate boxes which allows simulation of a plane wave with two Floquet modes that represent the incident horizontally and vertically polarized electromagnetic plane

\footnotetext{
1 https://www.ansys.com/products/electronics/ansys-hfss
} 

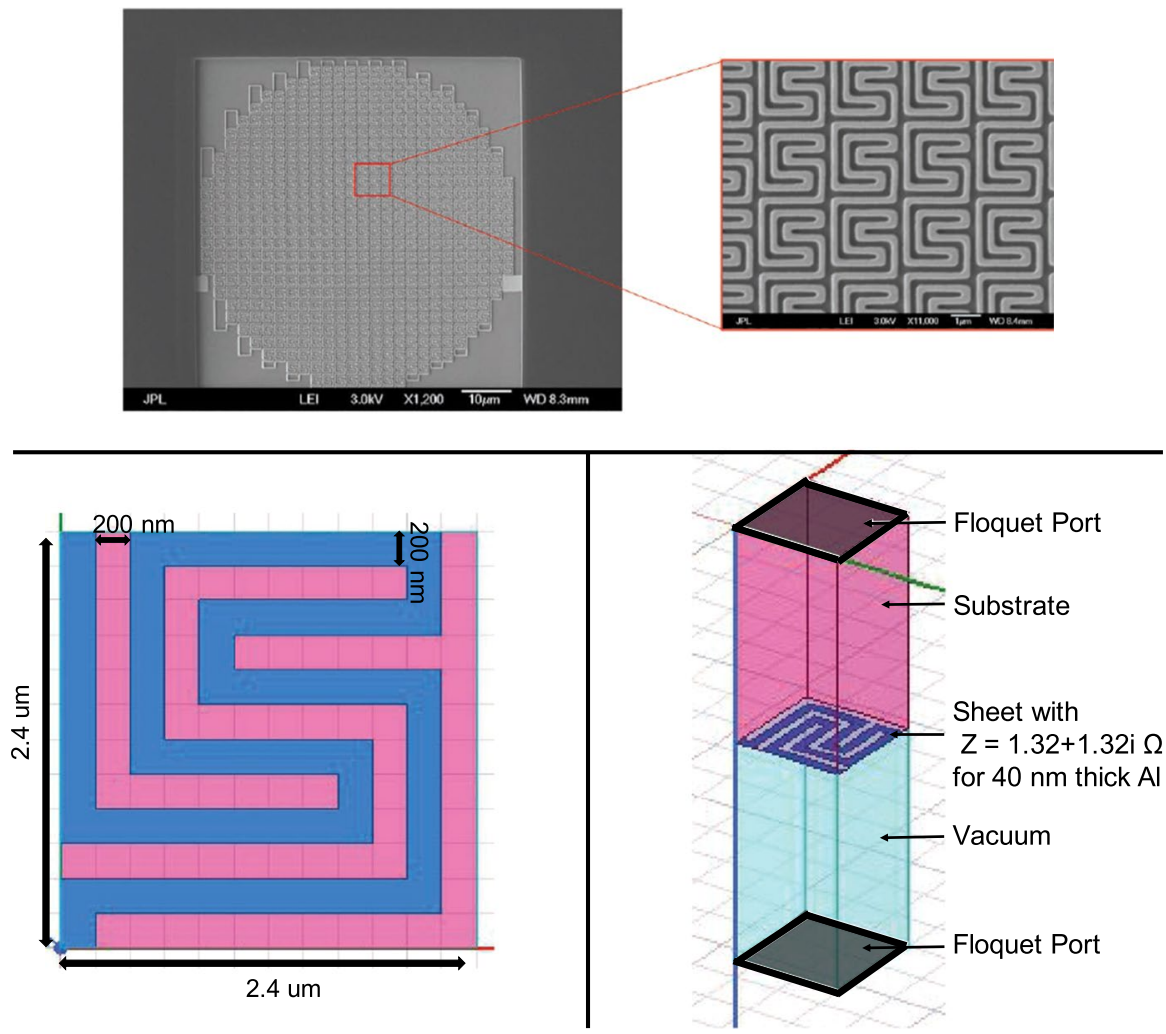

Fig. 1 Top Photograph of the inductor portion of a $10 \mu \mathrm{m}$ KID. The unit cell shown in Fig. 1 Bottom Left is repeated to cover the entire absorber area, which has a circular envelope with a diameter of $60 \mu \mathrm{m}$. Bottom Left short-meander unit cell geometry. The blue represents $\mathrm{Al}$ with $Z=(1.32+1.32 i) \Omega$ and the pink represents the Si substrate. The material of the microlens array that couples radiation to the KIDs will be made of the same material as the substrate. The total unit cell size depicted is $2.4 \times 2.4 \mu \mathrm{m}$, but will vary according to wavelength and substrate material. Bottom Right 3D model of HFSS unit cell simulation. The plane wave travels from the top Floquet port to the bottom Floquet Port. The vacuum and substrate box heights are not to scale. The heights were adjusted to show where the Floquet ports are assigned. The actual box height is equal to two times the longest wavelength of the simulation frequency sweep (Color figure online)

waves. HFSS achieves the periodic grid condition by assigning linked boundaries between parallel walls of the vacuum and substrate boxes of the unit cell. The linked boundaries enforce the parallel walls to have the same fields. The aluminum (blue meander in Fig. 1 Bottom) was modeled with sheets of impedance set to that of 40-nmthick aluminum exposed to $30 \mathrm{THz}$ electromagnetic radiation on a substrate (Fig. 1 Bottom). The impedance was determined by the following equation:

$$
Z_{s}=\frac{1+i}{\delta \sigma} \operatorname{coth}\left(\frac{1+i}{\delta} t\right),
$$



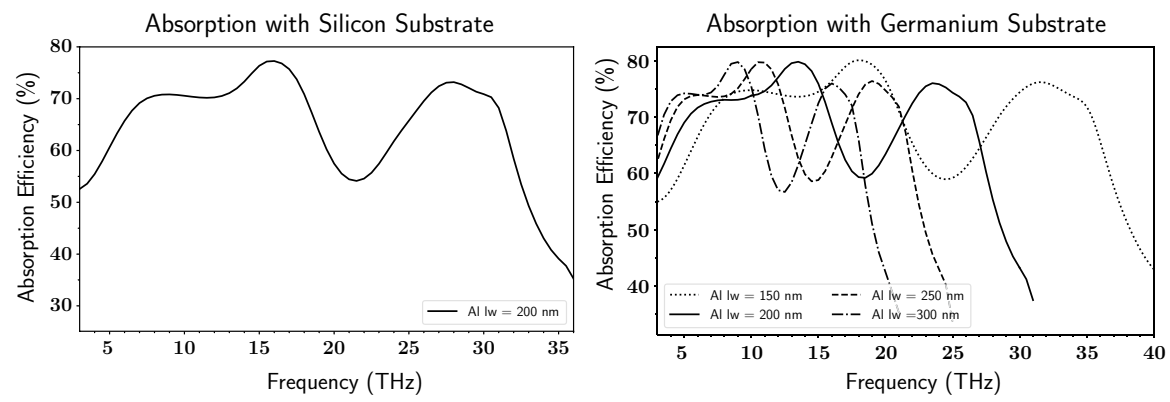

Fig. 2 HFSS-simulated absorption efficiency of short meander ( $1 \mathrm{w}=$ aluminum line width). Left Absorption efficiency of about $73 \%$ near $30 \mathrm{THz}$ on Si substrate. Right Absorption efficiency shifts to lower frequencies (greater wavelength) as $1 \mathrm{w}$ is increased on Ge substrate. Absorption efficiency varies from 16-32 THz, showing that the detector is capable of absorbing different wavelengths by adjusting the aluminum line width and unit cell size
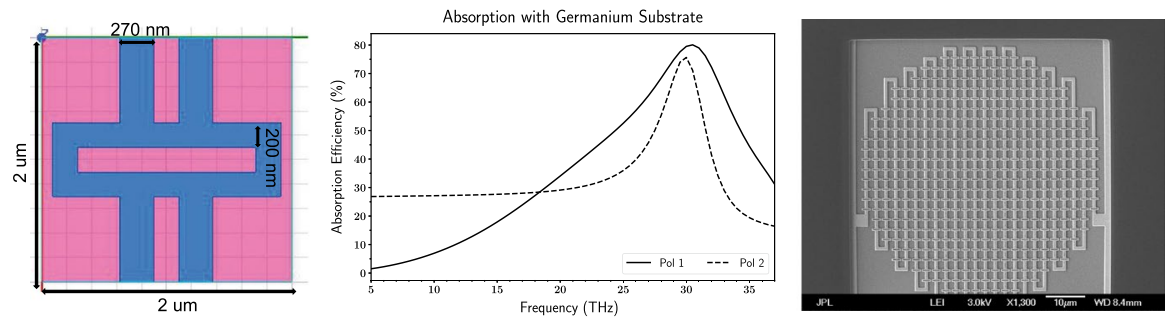

Fig. 3 Left Dual polarization $10 \mu \mathrm{m}$ KID inductor geometry. HFSS simulations of this geometry were performed the same as the short meander (Fig. 1 Bottom right) to approximate the repeated geometry across the circular profile of the inductor. The blue represents $\mathrm{Al}$ with $Z=(1.32+1.32 i) \Omega$ and the pink represents the Si substrate. Middle HFSS-simulated absorption efficiency of $75-80 \%$ near $30 \mathrm{THz}$ in both polarizations. These absorption profiles match GEP bandwidths better than those in Fig. 2 [4]. Right Photograph of the inductor portion of a $10 \mu \mathrm{m}$ KID. The unit cell shown in the left panel is repeated to cover the entire absorber area, which has a circular envelope with a diameter of $60 \mu \mathrm{m}$. Current flows along the vertical direction of the meander (Color figure online)

where $\delta=11.14 \mathrm{~nm}$ is the skin depth, $\sigma=6.87 \times 10^{7} \Omega^{-1} \mathrm{~m}^{-1}$ is the conductivity, and $t$ is the thickness of the aluminum [2]. Between the different geometries investigated, two showed promising results: one was a short meander (Fig. 1 Bottom Left) and the other a dual polarization geometry (Fig. 3 Left).

The $2.4 \times 2.4 \mu \mathrm{m}$ unit cell with the short-meander geometry shown in Fig. 1 Bottom Left has aluminum line widths and gaps of $200 \mathrm{~nm}$. The vertical lines couples to vertically polarized light, and the horizontal portion of the meander increases the resistance per unit length, enabling high absorption efficiency. This geometry was chosen because of its high HFSS-simulated absorption efficiency at $30 \mathrm{THz}$ (Fig. 2 Left). With aluminum line widths of $200 \mathrm{~nm}$ and on a silicon (Si) substrate, the absorption efficiency at $30 \mathrm{THz}$ is near $73 \%$. Since the absorption profile is broad, spectral bands will be defined with band-pass filters. The short meander was also simulated on a germanium (Ge) substrate (Fig. 2 Right), because Ge has better 
transmittance than Si from 10-20 $\mu \mathrm{m}$ [3]. The Ge simulations show that increasing the line width which in turn increases the unit cell size shifts the absorption to longer wavelengths. This means different geometries are not needed for different wavelength absorption. However, for absorption at $30 \mathrm{THz}$ on $\mathrm{Ge}$, the aluminum line widths need to be less than $200 \mathrm{~nm}$ (Fig. 2 Right). Without e-beam lithography fabrication of a device with such thin line widths is difficult, so a different geometry was explored, the dual polarization geometry shown in Fig. 3 Left.

The dual polarization geometry absorbs in both vertical and horizontal polarizations (Fig. 3 Middle). Another nice feature of this design is a higher absorption efficiency of $75-80 \%$ at $30 \mathrm{THz}$. It absorbs at $30 \mathrm{THz}$ with vertical line widths of $270 \mathrm{~nm}$, horizontal line widths of $200 \mathrm{~nm}$, and $200 \mathrm{~nm}$ gaps in a $2 \times 2 \mu \mathrm{m}$ unit cell. Since the unit cell size does not need to scale with aluminum line width there is more room to explore how changing parameters such as line and gap thickness changes the absorption peak profile. This absorber would be useful for observatories that need detectors which absorb in both polarizations.

\section{Preliminary Measurements}

A test device with the short-meander design and 40-nm-thick aluminum was fabricated at the Microdevices Laboratory at JPL, on a Si substrate (Fig. 1 Top). Each KID consists of a lithographically patterned absorbing inductive section connected to a large interdigitated capacitor and coupled to the feedline with small interdigitated capacitors [5]. The inductor portion of the lumped-element KIDs consists of the short-meander pattern shown in Fig. 1 Bottom Left repeated across a circular profile with a diameter of $60 \mu \mathrm{m}$ for effective optical coupling to a microlens array. Initial testing showed over $94 \%$ yield for resonant frequencies from 1.4-2 GHz with high internal Q-factors of over 200,000. We performed preliminary measurements to estimate the $T_{\mathrm{c}}$ of the device. Evidence of TLS noise was found.

\section{1 $T_{\mathrm{c}}$ Measurements}

The critical temperature of the test device was estimated by obtaining the fractional frequency shift of a detector's resonance with increasing temperature from 25 to $400 \mathrm{mK}$ (Fig. 4 Left). The data were fit to the following equation for fractional frequency shift:

$$
X=\frac{f_{\mathrm{r}}(T)-f_{\mathrm{r}}\left(T_{\text {base }}\right)}{f_{\mathrm{r}}\left(T_{\text {base }}\right)}=0.5 \alpha\left(\frac{\sigma_{2}-\max \left(\sigma_{2}\right)}{\max \left(\sigma_{2}\right)}\right),
$$

where $\sigma_{2}=\frac{\pi \Delta_{0}}{h f_{\mathrm{r}}}\left(1-\sqrt{\frac{2 \pi k_{\mathrm{b}} T}{\Delta_{0}}} e^{\frac{-\Delta_{0}}{k_{\mathrm{b}} T}}-2 e^{\frac{-\Delta_{0}}{k_{\mathrm{b}} T}} J_{0}\left(\frac{h f}{2 k_{\mathrm{b}} T}\right)\right), f_{\mathrm{r}}$ is the resonant frequency, $T$ is the temperature, $T_{\text {base }}$ is the initial temperature, and $k_{\mathrm{B}}$ is Boltzmann's constant [6, 7]. The best fit was determined using the $\chi^{2}$ minimization method with $\alpha$ (kinetic inductance fraction) and $\Delta_{0}$ (band gap energy) as the estimate parameters. $T_{\mathrm{c}}$ was 

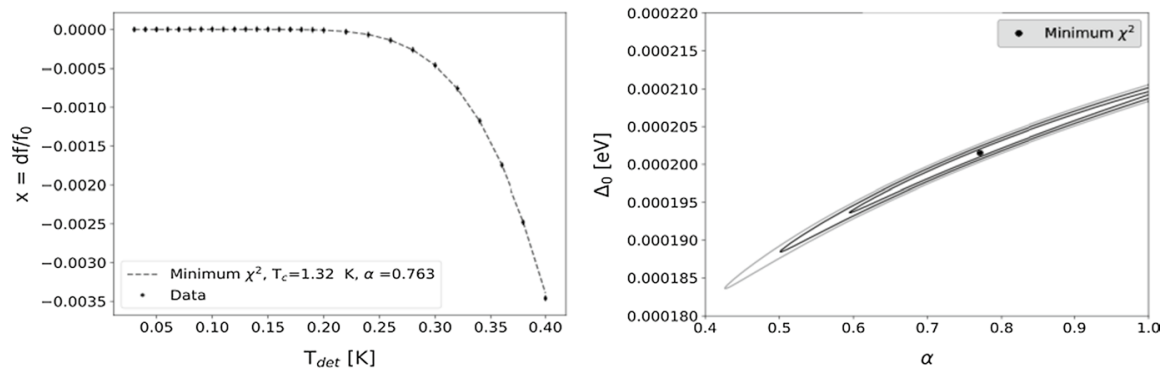

Fig. 4 Left Fractional frequency shift as a function of temperature. The dashed line represents the best fit model. Right $\Delta \chi^{2}$ contour plots of $1 \sigma, 2 \sigma$, and $3 \sigma$ uncertainties in the parameters. The $1 \sigma$ bounds were used to assign an error to $T_{\mathrm{c}}$, which yielded $T_{\mathrm{c}}=(1.32 \pm 0.05) \mathrm{K}$
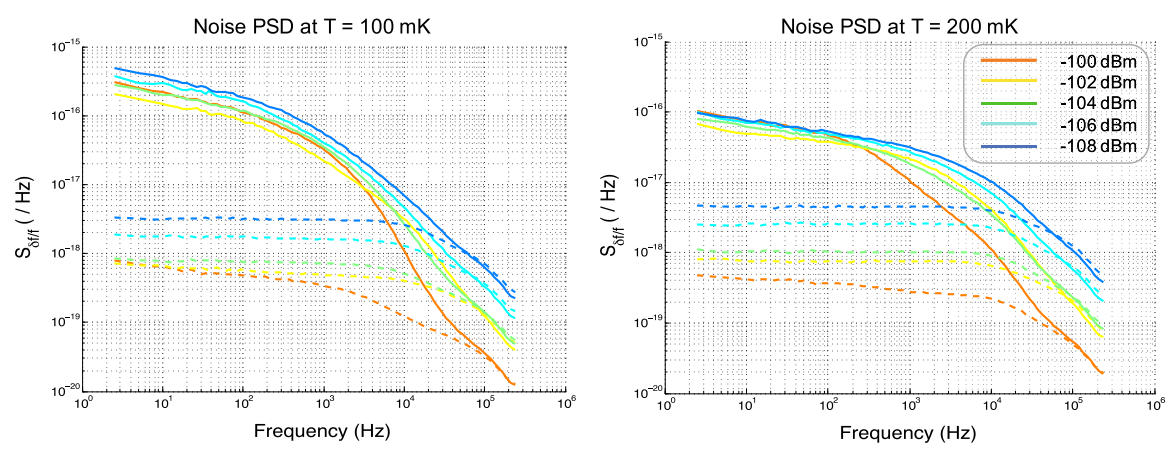

Fig. 5 Plots showing evidence of TLS noise. Dashed lines $=$ amplitude noise, solid lines $=$ phase noise. As the driving power decreases, the noise increases (driving power starts at $-100 \mathrm{dBm}$ and is attenuated by $2 \mathrm{~dB}$ with each curve). At higher temperature $(200 \mathrm{mK})$, the noise decreases (Color figure online)

then estimated using the relation $\Delta_{0}=1.764 k_{\mathrm{B}} T_{\mathrm{c}}$, yielding $T_{\mathrm{c}}=(1.32 \pm 0.05) \mathrm{K}$. This result is compatible with the $T_{\mathrm{c}}$ of aluminum found in the literature $[8,9]$.

\subsection{TLS Noise}

TLS noise is caused by an ensemble of switching dipoles in the substrate that fluctuates the dielectric constant, thereby altering the capacitance of the KIDs [10]. Evidence of TLS noise can be detected from the noise power spectral densities (PSDs) [7]. As the temperature is increased, TLS noise should decrease, and as the driving power increases, TLS noise should also decrease, because the twolevel systems become saturated in the upper state [7]. Figure 5 shows noise PSDs at $T=100 \mathrm{mK}$ and $T=200 \mathrm{mK}$. We see there is an evident decrease in phase noise at the higher temperature. With drive power attenuation, the noise increases which is another characteristic of TLS noise shown in Fig. 5. 


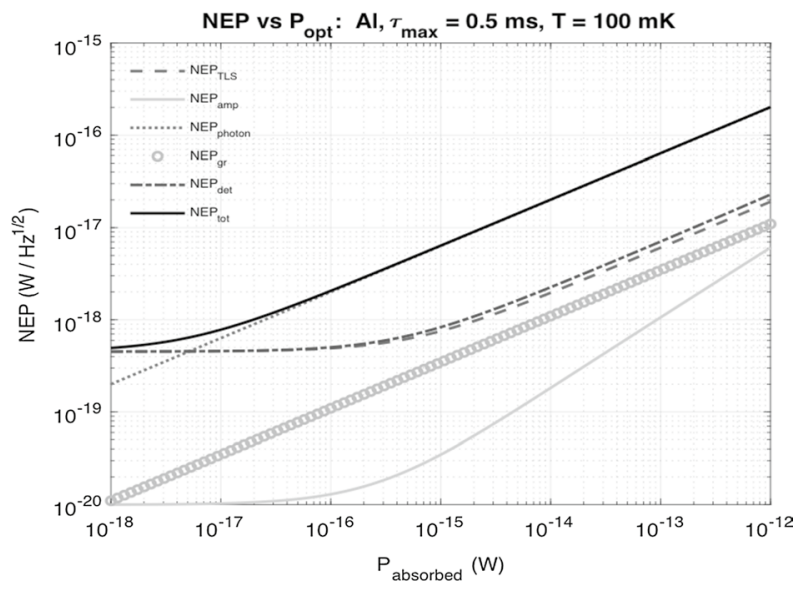

Fig. 6 Theoretical NEP plot for GEP

\section{GEP Theoretical NEP}

Since we have not yet done optical testing, we provide a theoretical NEP calculation for GEP that takes into account the measured TLS noise and estimated $T_{\mathrm{c}}$ and $\alpha$ from Sect. 3. The total NEP of the test device is calculated by adding all NEP contributions in quadrature, $\mathrm{NEP}_{\text {tot }}^{2}=\mathrm{NEP}_{\mathrm{TLS}}^{2}+\mathrm{NEP}_{\mathrm{amp}}^{2}+\mathrm{NEP}_{\text {photon }}^{2}+\mathrm{NEP}_{\mathrm{GR}}^{2}[6,7]$. Since we have not measured quasiparticle lifetimes $\left(\tau_{\mathrm{qp}}\right)$, an assumption was made based on previously measured 40-nm Al KIDs that $\tau_{\mathrm{qp}}=500 \mu s$ [11]. The operating temperature will be $T=100 \mathrm{mK}$ for GEP. At $10 \mu \mathrm{m}$, we expect the photon loading to be $2.5 \times 10^{-15} \mathrm{~W}$. This corresponds to a theoretical NEP of $1 \times 10^{-17} \mathrm{~W} / \sqrt{\mathrm{Hz}}$ (Fig. 6).

\section{Summary and Future Work}

We have designed aluminum KIDs on $\mathrm{Si}$ and Ge substrates with meanders for tuning resistivity that yield simulated absorption efficiencies of $73-80 \%$ at $10 \mu \mathrm{m}$. The absorption can be shifted in wavelength by adjusting the aluminum line widths and unit cell sizes of the geometry repeated across the inductor of the KID. A test device with $10 \mu \mathrm{m}$ aluminum KIDs was fabricated, from which $T_{\mathrm{c}}=(1.32 \pm 0.05) \mathrm{K}$ was estimated and evidence of TLS noise was found. We also present a theoretical NEP for GEP at $10 \mu \mathrm{m}$ that takes into account the estimated $T_{\mathrm{c}}$ and measured TLS noise from the fabricated device.

Our next steps are to investigate zinc sulfide as an anti-reflection coating in the HFSS simulations to increase absorption efficiency [3], couple the KIDs with the short-meander geometry to a microlens array for optical testing, fabricate a device with the dual polarization geometry, and measure $\tau_{\mathrm{qp}}$ and NEPs for both. We will also begin development of $30 \mu \mathrm{m}$ aluminum KIDs. 
Funding Funding was provided by National Aeronautics and Space Administration (Grant No. 80NSSC19K0489).

Open Access This article is licensed under a Creative Commons Attribution 4.0 International License, which permits use, sharing, adaptation, distribution and reproduction in any medium or format, as long as you give appropriate credit to the original author(s) and the source, provide a link to the Creative Commons licence, and indicate if changes were made. The images or other third party material in this article are included in the article's Creative Commons licence, unless indicated otherwise in a credit line to the material. If material is not included in the article's Creative Commons licence and your intended use is not permitted by statutory regulation or exceeds the permitted use, you will need to obtain permission directly from the copyright holder. To view a copy of this licence, visit http://creativecommons.org/licen ses/by/4.0/.

\section{References}

1. A. Lger, L. d'Hendecourt, D. Defourneau, Physics of IR emission by interstellar PAH molecules. Astron. Astrophys. 216, 148-164 (1989)

2. R.E. Matick, Transmission Lines for Digital and Communication Networks: An Introduction to Transmission Lines High-Frequency and High-Speed Pulse Characteristics and Applications (IEEE Press, London, 1995)

3. J.T. Cox, G. Hass, Antireflection coatings for germanium and silicon in the infrared. J. Opt. Soc. Am. 48(10), 677 (1958). https://doi.org/10.1364/JOSA.72.000027

4. J. Glenn et al., The Galaxy Evolution Probe: a concept for a mid and far-infrared space observatory. Space Telescopes and Instrumentation 2018: Optical, Infrared, and Millimeter Wave. Vol. 10698. International Society for Optics and Photonics (2018). https://doi.org/10.1117/12.2314076

5. J. Glenn et al., Low-volume aluminum and aluminum/titanium nitride bilayer lumped-element kinetic inductance detectors for far-infrared astronomy. Millimeter, Submillimeter, and Far-Infrared Detectors and Instrumentation for Astronomy VIII. Vol. 9914. International Society for Optics and Photonics (2016). https://doi.org/10.1117/12.2233649

6. J. Gao, The physics of superconducting microwave resonators. Diss. California Institute of Technology (2008)

7. J. Zmuidzinas, Superconducting microresonators: physics and applications. Annu. Rev. Condens. Matter Phys. 3(1), 169-214 (2012)

8. L. Cardani et al., Energy resolution and efficiency of phonon-mediated kinetic inductance detectors for light detection. Appl. Phys. Lett. 107(9), 093508 (2015). https://doi.org/10.1063/1.4929977

9. A. Paiella et al., Development of lumped element kinetic inductance detectors for the w-band. J. Low Temp. Phys. 184(1-2), 97-102 (2016). https://doi.org/10.1007/s10909-015-1470-z

10. O. Noroozian et al., Twolevel system noise reduction for microwave kinetic inductance detectors. In: AIP Conference Proceedings, Vol. 1185. No. 1. AIP (2009). https://doi.org/10.1063/1.3292302

11. A. Fyhrie et al., Progress towards ultra sensitive KIDs for future far-infrared missions: a focus on recombination times. In: Millimeter, Submillimeter, and Far-Infrared Detectors and Instrumentation for Astronomy IX. Vol. 10708. International Society for Optics and Photonics (2018). https://doi. org/10.1117/12.2312867

Publisher's Note Springer Nature remains neutral with regard to jurisdictional claims in published maps and institutional affiliations. 